\title{
Primary Neuroendocrine Tumor of the Testis Masquerading as Germ Cell Tumor - A Case Report
}

\author{
Sulagna Manna, Suma Mysore Narayana, C. S. Premalata
}

\section{ABSTRACT}

\begin{abstract}
Primary pure neuroendocrine tumors of the testis are rare with a high malignant potential requiring long-term follow-up. Only limited number of studies are available in the literature.
\end{abstract}

In the present study, we describe a case report of a 31 year old young man who presented with a left testicular swelling, initially misdiagnosed as seminoma. Later, after correlating with the morphology and immunohistochemistry the diagnosis of neuroendocrine tumor of testis was concluded.

Neuroendocrine tumors cases should be kept in long term follow up, to prevent relapse and recurrence.

Keywords: Neuroendocrine tumors, Chromogranin, Synaptophysin, Seminoma, Immunohistochemistry.

\section{INTRODUCTION}

Testicular germ cell tumors, seminoma and nonseminomas constitute more than $90 \%$ of all type II germ cell tumors. On the contrary, testicular neuroendocrine tumors are extremely rare variety of tumors and accounts for $<1 \%$ of overall testicular neoplasms [1]. These tumors can occur as primary tumors or as metastases [2]. These patients typically present between the second and ninth decades of life, with an average age at presentation of 46 years. These cases can be misdiagnosed, hence the differential diagnosis of metastatic neuroendocrine carcinoma, testicular teratoma with neuroendocrine tumor, seminoma, sertoli cell tumor and granulosa cell tumor should be kept in mind [3], [4].

Here, we report a case of primary neuroendocrine tumor of the testis which was misdiagnosed as seminoma.

\section{CASE REPORT}

A 31 years old man, moderately built and nourished was referred from the primary centre after orchidectomy. On asking for the history patient revealed, he had presented to a primary centre with left scrotal swelling for one year. There was no other significant past history and family history of testicular cancer. The records mentioned that, on external examination there was a bulge in the scrotal sac. The overlying skin was pinchable. There was no ulceration noted. The CT abdomen and pelvis revealed well defined heterogeneously enhancing mass lesion measuring
Published Online: November 18, 2020 ISSN: 2593-8339

DOI: $10.24018 /$ ejmed.2020.2.6.518

\section{Dr Sulagna Manna *}

MD, DNB, Fellow in Tumor Pathology, Department of Pathology, Kidwa Memorial Institute of Oncology, India.

(e-mail: sulagna.manna ${ }^{\circledR}$ gmail.com)

Dr Suma Mysore Narayana

MD, Associate Professor, Department of Pathology, Kidwai Memorial Institute of Oncology, India.

Dr C.S. Premalata

MD, Professor and Head, Department of Pathology, Kidwai Memorial Institute of Oncology, India.

*Corresponding Author
$5.2 \times 5.0 \times 3.0 \mathrm{~cm}$ in the left testis. Intralesional foci of small, stippled areas of calcification were noted. With these findings an impression of malignant neoplasm of the left testis was made. Lung was normal on CT thorax. His right testis was normal.

The quantitative serological markers performed were as follows:

Beta-HCG <2.3 (normal <5.0 U/L), AFP 1.35 (normal $<15.0$ micro $\mathrm{g} / \mathrm{L}$ ). All the markers were within normal limits.

The patient underwent surgery for exploration and radical orchiectomy. The histomorphological diagnosis of classic seminoma of left testis, high inguinal orchidectomy was made. At this point the patient was referred to Kidwai Memorial Institute of Oncology, Bangalore, India for further management, as our institute is a tertiary cancer care centre. The patient submitted paraffin blocks, slides, and partially grossed specimen at our institute for second opinion.

\section{OBSERVATIONS}

\section{A. Macroscopic Findings}

We received already grossed specimen of orchidectomy comprising of spermatic cord measuring $5 \mathrm{~cm}$ in length. The testis measured $7 \mathrm{X} 4.5 \mathrm{X} 3 \mathrm{~cm}$ and epididymis measured $2.3 \mathrm{X} 1.3 \mathrm{X} 2 \mathrm{~cm}$. External surface of testis appeared enlarged. On serial slicing the entire testis was replaced by a grey white homogenous tumor measuring $6 \times 5 \times 3 \mathrm{~cm}$ with focal central haemorrhagic area measuring $1 \mathrm{~cm}$ (Fig. 1). A small rim of unremarkable testis was seen at the upper pole. It was soft to 
firm in consistency. Tumor was confined to the testis. Tunica albugenia and tunica vaginalis was unremarkable. Epididymis was grossly unremarkable.

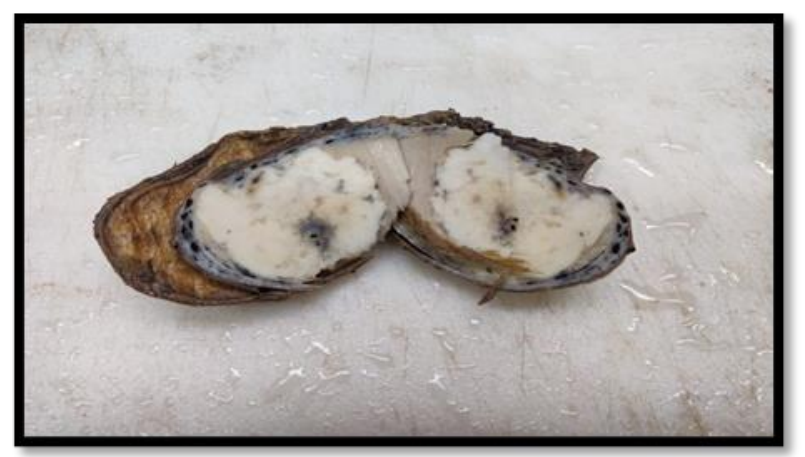

Fig. 1. Shows a well circumscribed grey white tumor rimmed by normal testis with areas of necrosis and haemorrhage.

\section{B. Microscopic Findings}

Multiple sections from the testicular tumor displayed a neoplasm composed of tumor cells arranged in lobules of various sizes separated by broad hyaline septae. The individual tumor cells were arranged in cords, trabeculae, acini, and clusters. The cells were round, regular, exhibited scant eosinophilic to clear cytoplasm, fine nuclear chromatin, and some of them displaying conspicuous nucleolus. Lymphocytic infiltrate was minimal. Mitosis was infrequent (8-10/10 hpf). Small foci of necrosis were noted. No Germ cell neoplasia in situ (GCNIS) was noted in the adjacent testicular tissue. The spermatic cord and tunica albuginea were free of tumor. On extensive sampling, no other teratomatous component was present (Fig. 2).

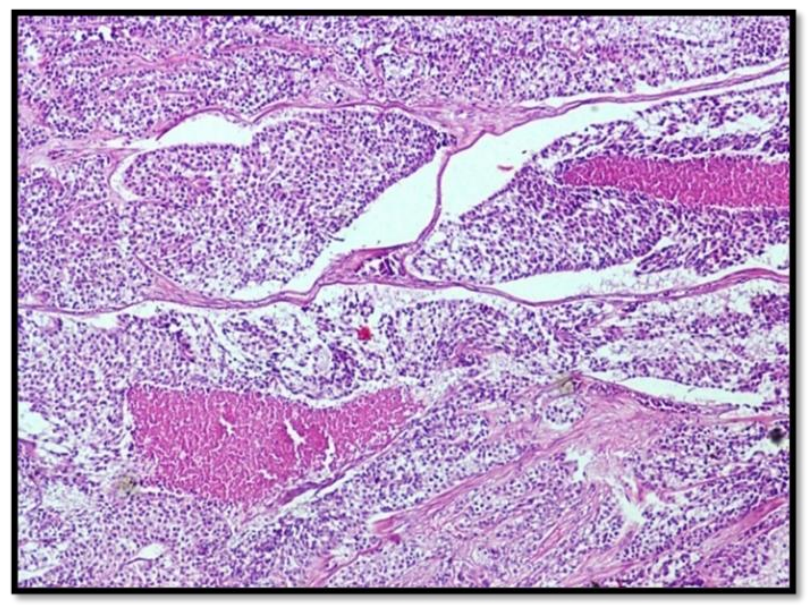

Fig. 2. Histopathological examination of the tumor: Low power view of the monotonous population of the tumor cells in cords, trabeculae, acini, and clusters. The cells are round to oval with moderate to scant cytoplasm and have fine chromatin.

\section{Immunohistochemistry Findings}

The neoplastic cells were focally positive for synaptophysin (Fig. 4) and negative for chromograninA (Fig. 6). They showed strong cytoplasmic CD56 positivity (Fig. 3). Ki-67 proliferation index was $15-18 \%$ (Fig. 5). The germ cell tumor markers like SALL4, PLAP, OCT3/4, and Glypican3 were negative. The sex cord tumor markers like inhibin and calretinin were negative. They were also negative for CK20, CK7, EMA, CD99, LCA, NKX2.2, FLI1, WT1, desmin, D240 and mesothelin.

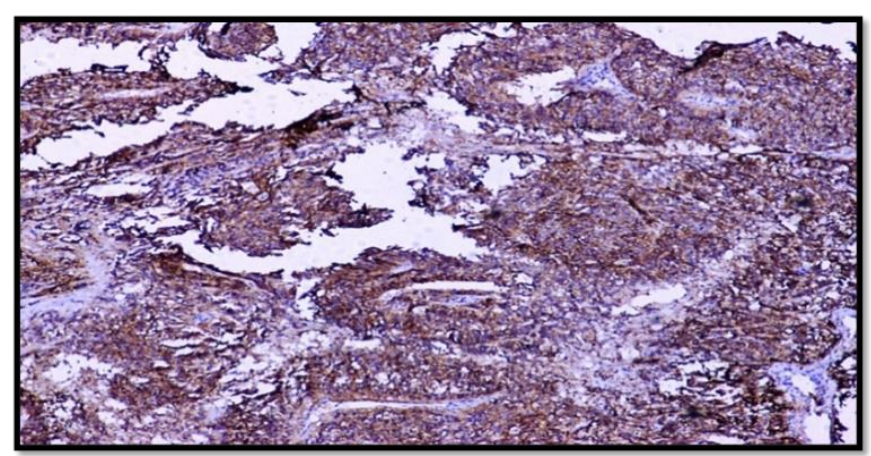

Fig. 3. Immunohistochemical Staining; Tumor cells show strong cytoplasmic staining by CD56.

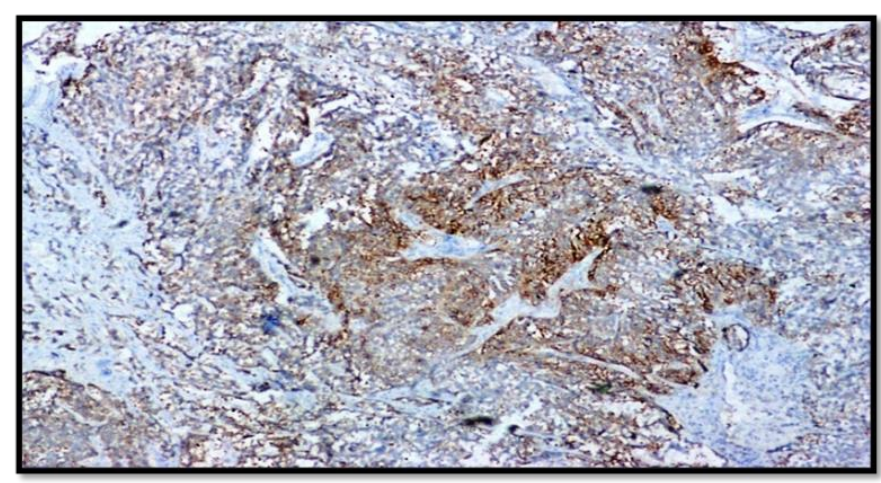

Fig. 4. Tumor cells show focal cytoplasmic granular positivity by synaptophysin.

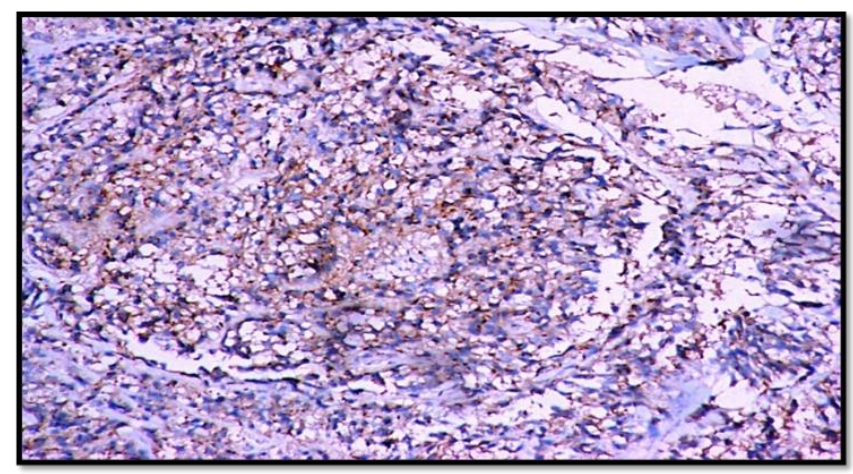

Fig. 5. Ki-67 proliferation index is $15-18 \%$.

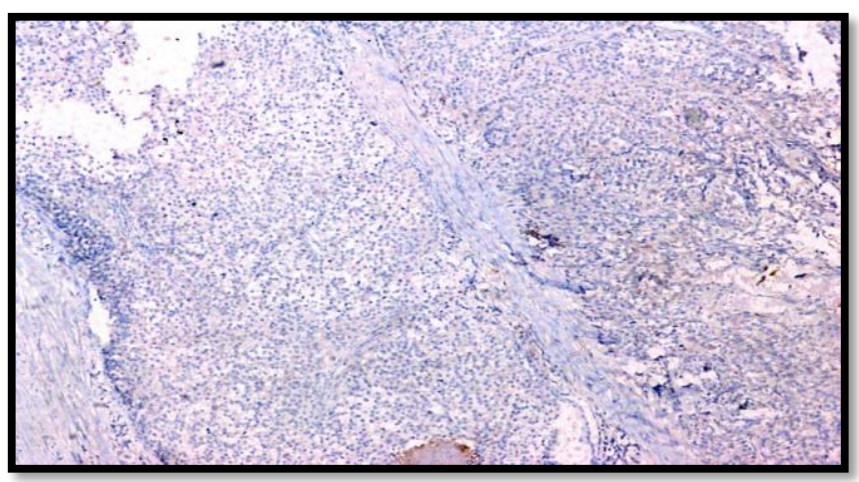

Fig. 6. Tumor cells were negative for Chromogranin A

The primary diagnosis of Neuroendocrine tumor Grade 2, was offered based on the morphological features and immunohistochemical study. Since primary Neuroendocrine tumors of the testis is very rare we suggested systemic workup for any other primary neoplasm. Further CT scan and PET scan did not reveal any focus of tumor elsewhere in the 
body. A final diagnosis of Primary pure neuroendocrine tumor, Grade 2 was made. The patient is now kept on follow up.

\section{DiscUSSION}

Neuroendocrine neoplasms occur most commonly in the gastrointestinal tract $(65 \%)$ followed by respiratory tract $(25 \%)$ and are very rarely found in the testis, which could be primary or metastatic which accounts to be less than $1 \%$ [2], [3].

Most cases of the testicular neuroendocrine tumor ranges from 10 to 83 years of age. Most of the patients present with unilateral painless testicular mass. $16 \%$ of patients present with symptoms of neuroendocrine tumor syndrome. $11 \%$ of primary testicular neuroendocrine tumors are associated with metastasis [3].

The primary testicular neuroendocrine tumor can be further divided into 1) primary pure testicular neuroendocrine tumor and 2) secondary neuroendocrine malignancies. Secondary neuroendocrine carcinomas can metastasize from pulmonary or gastrointestinal neuroendocrine carcinomas and can also be associated with teratomas and dermoid cysts. Metastatic workup of the testicular neuroendocrine tumors are extremely necessary. Neuroendocrine tumor of the testis should always be distinguished from metastatic neuroendocrine carcinoma, testicular teratoma with neuroendocrine tumor, seminoma, sertoli cell tumor and granulosa cell tumor [4].

\section{A. Metastatic Neuroendocrine Carcinoma}

The primary lesion commonly originates from the lung or digestive tract, and occasionally from the urogenital tract, adrenal gland, or prostate. Testicular metastatic neuroendocrine carcinoma generally involves both testes and is associated with multifocal and vascular invasion. In contrast, patients with primary neuroendocrine tumors usually present with a long history of unilateral testicular swelling or lump, and typically do not have tumors of other tissues or organs [4].

\section{B. Testicular Teratoma with Neuroendocrine Tumor}

The composition of this tumor is complicated and includes both teratoma component and neuroendocrine tumor component. Previously primary neuroendocrine tumor with testicular teratoma was called testicular mixed carcinoid; these tumors account for approximately $20 \%$ of primary testicular neuroendocrine tumors. The tumor is encapsulated with a thin layer of fibrous connective tissue, and contains tissues such as sebaceous glands, sweat glands and cartilage, which is lined with stratified squamous epithelium. The tumor cells of neuroendocrine tumor are of a consistent size and shape, form irregular islands, trabeculae or small adenoid arrangements and show an invasive growth pattern. On immunohistochemical analysis, the tumor cells are positive for CK, CD56, Synaptophysin, Chromogranin A and S-100 [4].

\section{Seminoma}

Seminoma is a germ cell tumor of the testis. On microscopic examination, the tumor cells can be seen to be arranged in lobules, divided by thins septae. The interstitium shows lymphocytic infiltration or lymphoid follicles. The tumor cells are round or polygonal, with well-defined boundaries, transparent and mild eosinophilic granular cytoplasm containing glycogen and lipid. Immunohistochemical analysis shows that the tumor cells are positive for OCT3/4, CD117, PLAP, D2-40 and negative for CD56, Synaptophysin and Chromogranin A [4], [5].

\section{Sertoli Cell Tumor}

Sertoli cell tumor is relatively rare, accounting for $1 \%$ of testicular tumors. Occurs at any age, but typically occurs in middle-aged persons. The tumor appears as a solid, white, or grey nodule, with a diameter of less than $3 \mathrm{~cm}$. On microscopy, the tumor cells are seen to be arranged in a solid nest, solid tubular, or cribriform pattern. The interstitium shows dense fibrous stroma often accompanied with hyaline degeneration. Immunohistochemical analysis shows the tumor cells positive for vimentin and inhibin and negative for CK, CD56, Synaptophysin and Chromogranin A [4].

\section{E. Granulosa Cell Tumor}

Adult testicular granulosa cell tumor is quite rare and tends to occur in persons aged 16-67 years. This tumor is associated with high oestrogen levels, which usually cause breast development. Malignant tumors are often greater than $7 \mathrm{~cm}$ in diameter, and show vascular infiltration, haemorrhage, and necrosis. Microscopic examination shows that the tumor cells are arranged in follicle, nests or sarcomatoid arrangement. The follicles often have Call- Exner small body structure [4].

The neuroendocrine neoplasms have been defined into 3 groups by WHO/ European Neuroendocrine Tumor Society (ENETS). The classification is based on the immunostaining of Ki-67 or mitotic count - Neuroendocrine Tumors NET G1: Ki-67 <3\%, NET G2: Ki-67 3-20\%, NET G3: Ki-67 > 20\% [5], [6].

Diagnosis of the primary neuroendocrine tumor is made on the basis of clinical, ultrasonographic, histopathological and immunohistochemical findings. Surgical resection is the main modality of the treatment with postoperative radiation and chemotherapy if required. A close follow-up should be done. The nature, size, site of the tumor, the depth of invasion, and lymph node metastasis, determines the extent and manner of excision of the tumor [7].

Primary neuroendocrine tumor (G1) is diagnosed based on clinical, ultrasonographic, histopathological and immunohistochemical findings. In the case of neuroendocrine tumor (G1), surgical resection with close follow-up is the treatment of choice [10]. The extent and manner of excision depend on the nature, size, and site of the tumor, the depth of invasion, lymph node metastasis, etc. [11], [12]. The survival rate of patients with primary testicular neuroendocrine tumor with lymph node and lung metastases can be increased with chemotherapy (cisplatin and etoposide; ifosfamide, epirubicin and octreotide), radiation therapy, etc. [8]. Some authors have reported that cisplatin-based chemotherapy regimens are the most useful to prevent tumor metastasis. Tumor size and carcinoid syndrome are features associated with a malignant course. Patients with carcinoid syndrome have a poor prognosis [9], [10]. 


\section{CONCLUSION}

Primary testicular neuroendocrine tumor is very rare. It is always a necessity to rule out the other germ cell neoplasm of testis. Patients should always be kept on follow up to prevent metastasis and recurrences. Our patient underwent surgery and is kept on close follow up. He is alive, doing well, and regularly attends follow-up appointments. This case is reported due to its rarity.

\section{ACKNOWLEDGMENT}

The authors express their gratitude to the authority, Kidwai Memorial Institute of Oncology Bangalore, INDIA, and the patient and technical staffs for cooperating in the work.

\section{REFERENCES}

[1] C. Lu, Z. Zhang, Y. Jiang, Z. Yang, Q. Yang, D. Liao et al., Primary pure carcinoid tumors of the testis: Clinicopathological and immunophenotypical characteristics of 11 cases. Oncology Letters, 2015, Vol. 9(5), pp. 2017-2022.

[2] M. Al-Shraim, A. Alhadi, A. Al-Aown, F. Fooshang, R. Eid, S. Alsharif, Primary neuroendocrine tumor of the testis, Urology Annals, 2014, Vol.6(2), pp. 173.

[3] D. Heimann, Primary Neuroendocrine Tumor (Carcinoid Tumor) of the Testis: A Case Report with Review of Literature. American Journal of Case Reports, 2015 Vol. 16, pp. 328-332.

[4] X. U. Han, L. Yu, J. Zheng, Primary Neuroendocrine Tumor: A Study of clinicopathological features. Int J Clin Exp Pathol, 2014, Vol. 7(4), pp. 1771-1776.

[5] Ghoreifi A, Liang T, Ballas L, Sherrod A, Djaladat H, Testicular Carcinoid Tumor in a Patient with Contralateral Classic Seminoma. Urology. 2020, Vol.141, pp32-34.

[6] J. Merino, Pure testicular carcinoid associated with intratubular germ cell neoplasia. Journal of Clinical Pathology, 2005 Vol. 58(12), pp. 1331-1333.

[7] M. Bolat, E. Akdeniz, F. Saltık, N. Sahinkaya, C. Moral, Primary Carcinoid Tumor of the Testis: Case Report. Urology Case Reports. 2015, Vol. 3(5), pp. 152-154.

[8] A. Petrossian, J. Habibi, D. Rapp, D. Ramnani, Primary Carcinoid Tumor of the Testis. Case Reports in Urology, 2015, Vol. 2015, pp. 1 4.

[9] R. Abou Zahr, K. Chalhoub, E. Mansour, C. Chouairy, G. Ghazal, J. Nohra, Primary Carcinoid Tumor of the Testis: A Case Report and Review of the Literature. Case Reports in Urology, 2018, Vol. 2018, pp.1-4.

[10] O. Stroosma, K. Delaere, Carcinoid tumours of the testis. BJU International. 2008, Vol.101 (9), pp 1101-1105.

[11] Lubana SS, Singh N, Chan HC, Heimann D, Primary neuroendocrine tumor (carcinoid tumor) of the testis: a case report with review of literature. The American Journal of Case Reports. 2015, Vol.16, pp 328-332.

[12] G. Kyriakopoulos, V. Mavroeidi, E. Chatzellis, G. Kaltsas, K. Alexandraki, Histopathological, immunohistochemical, genetic and molecular markers of neuroendocrine neoplasms. Annals of Translational Medicine, 2018, Vol. 6(12), pp. 252-252. 